\title{
Multi-particle suspension in a laminar flow agitated by a Rushton turbine
}

\author{
Chao Wang ${ }^{a, b}$, Li Zhang ${ }^{a, b}$, Zhipeng Li ${ }^{a, b,{ }^{*}}$, Zhengming Gao ${ }^{a, b,{ }^{*}}$, J.J. Derksen ${ }^{c}$ \\ a Beijing Advanced Innovation Center for Soft Matter Science and Engineering, Beijing University of Chemical Technology, Beijing 100029, China \\ b State Key Laboratory of Chemical Resource Engineering, School of Chemical Engineering, Beijing University of Chemical Technology, Beijing 100029, China \\ c School of Engineering, University of Aberdeen, Aberdeen AB24 3UE, UK
}

* Corresponding author. Tel.: +8610 64418267; fax: +8610 64449862. E-mail address: lizp@mail.buct.edu.cn (Zhipeng Li), gaozm@mail.buct.edu.cn (Zhengming Gao).

\begin{abstract}
Multi-particle suspension in a laminar stirred tank flow agitated by a standard Rushton turbine was investigated experimentally and numerically. In the experiments, the motion of the particles was measured by two high-speed cameras and was quantitatively analyzed. Two very stable particle patterns were observed. Strong flow is required to break these patterns because their formation hinders the particle lift-off process. The experimental trajectories and vertical velocities of the particles were measured, and highly reproducible results were found. Direct numerical simulations based on the lattice-Boltzmann method and the resolved particle model were performed to fully resolve the motion of the particles and the flow field. The influence of the friction coefficient and the subgrid lubrication force model on particle suspension was evaluated. Except for the random characteristics of particles interaction at the initial lift-off stage, the predicted trajectories and velocities of the particles are in good agreement with the experimental results. The pressure gradient around the particles is a key mechanism for the lift-off process.
\end{abstract}

Keywords: solid-liquid suspension; multiple particles; laminar flow; lattice-Boltzmann method; direct numerical simulation

\section{Highlights:}

- The motion of multiple particles was measured by using two high-speed cameras.

- Reproducible trajectories and velocities of the particles were obtained.

- Simulated trajectories and velocities of particles agree with experimental results. 


\section{Nomenclature}

$a \quad$ Particle radius [m]

$\mathrm{c}_{\mathrm{s}} \quad$ Sound speed $[\mathrm{m} / \mathrm{s}]$

C Off-bottom clearance of the Rushton turbine [m]

$d_{\mathrm{p}} \quad$ Particle Diameter [m]

$D \quad$ Impeller diameter [m]

$e \quad$ Restitution coefficient [-]

$\boldsymbol{F}_{\text {lub }} \quad$ Lubrication force $[\mathrm{N}]$

$F_{\text {susp }} \quad$ force suspending particles [N]

g Gravitational acceleration $\left[\mathrm{m} / \mathrm{s}^{2}\right]$

$H \quad$ Liquid height inside the tank [m]

n Unit normal vector [-]

$n_{\llcorner O} \quad$ Number of the particles being lifted off [-]

$n_{\text {total }}$ Total number of the particles [-]

$N \quad$ Impeller speed [revolution/s]

$N_{\mathrm{LO}} \quad$ Lift-off impeller speed [revolution/s]

Re Reynolds number based on impeller speed, $\operatorname{Re}=N D^{2} / v[-]$

$s_{0} \quad$ Distance between two solid surfaces below which the lubrication force switches on [m]

$s_{1} \quad$ Threshold below which the lubrication force saturates [m]

$t \quad$ Time $[\mathrm{s}]$

$T \quad$ Side length of the tank $[\mathrm{m}]$

$T_{\mathrm{m}} \quad$ Average temperature inside the tank $\left[{ }^{\circ} \mathrm{C}\right]$

u Fluid velocity vector $[\mathrm{m} / \mathrm{s}]$ 
$v_{\text {tip }} \quad$ Tip speed of the impeller, $v_{\text {tip }}=\pi N D[\mathrm{~m} / \mathrm{s}]$

$x, y, z$ Radial, tangential, and axial coordinates [m]

$\boldsymbol{\alpha}, \boldsymbol{\beta} \quad$ Relaxation factors [-]

$\Theta \quad$ Inertial Shields number, $\Theta=\rho N^{2} D^{2} / g \Delta \rho d_{\mathrm{p}}[-]$

$\mu \quad$ Friction coefficient [-]

$v \quad$ Kinematic viscosity of the silicone oil $\left[\mathrm{m}^{2} / \mathrm{s}\right]$

$\rho \quad$ Density of the silicone oil $\left[\mathrm{kg} / \mathrm{m}^{3}\right]$

$\rho_{\mathrm{p}} \quad$ Particle density $\left[\mathrm{kg} / \mathrm{m}^{3}\right]$

$\Delta \quad$ Grid spacing [lattice units]

$\Delta t \quad$ Time step [lattice units]

$\Delta \mathbf{u}_{i j} \quad$ Relative velocity vector, $\Delta \mathbf{u}_{i j}=\mathbf{u}_{\mathrm{pj}}-\mathbf{u}_{\mathrm{pi}}[\mathrm{m} / \mathrm{s}]$

$\Delta \rho \quad$ Density difference, $\Delta \rho=\rho_{\mathrm{p}}-\rho\left[\mathrm{kg} / \mathrm{m}^{3}\right]$

\section{Introduction}

Stirred tanks with solid-liquid mixtures are widely used in various industrial processes, such as petrochemical engineering, fine chemistry, reaction systems with solids catalyst, and crystallization processes. The critical impeller speed to suspend all solids is an important parameter for the design and optimization of the stirred tank; that is, with this speed, and thus minimum power input, all the solids can be suspended, ensuring maximum solid-liquid interfacial area. Extensive research has been carried out to determine the critical condition in stirred tanks. Zwietering (1958) pioneered the concept of just suspended speed $N_{\mathrm{js}}$ at which no particles rested on the bottom of the tank more than 1 or 2 second. As extensions of Zwietering's work, many researchers (Blais et al., 2017; Kee and Tan, 2002; Srinivasa and Jayanti, 2007) combined empirical correlations with numerical simulations to study solid-liquid suspension for different geometrical and operating conditions. 
Recently, various visualization experiments have been used to understand the suspension characteristics of particles in mixing tanks. Ayranci et al. (2012) used Particle Image Velocimetry (PIV) to investigate solids suspension mechanisms in a turbulent stirred tank flow. Li et al. (2017) also used PIV experiment to obtain the behavior of suspended particles with maximum $8 \%$ solids volume fraction. In the range of dense suspension, Carletti et al. (2014) used Electrical Resistance Tomography (ERT) to analyze the solids distribution by varying the solid loading in a stirred tank. A series of studies (Blais et al., 2016; Blais and Bertrand, 2017; Lassaigne et al., 2016) combined ERT with a Pressure Gauge Technique (PGT) to investigate how the particle properties, the viscosity of the liquid, and the configuration of the stirring system had effect on the just suspended speed $N_{\mathrm{js}}$ and on the homogeneity of the solids suspension in laminar and transitional regimes. The above works on the suspension of solid particles in stirred tank focus on the macroscopic kinetic behavior of a large number of particles. However, the particle-fluid and particle-particle interaction have not been probed in detail. For a better understanding of suspension processes, it is worthwhile exploring the dynamic behavior of a single particle or a small number of particles.

In solid-liquid sediment systems, the photographic technique as a general means to directly obtain particle motion has received extensive attention. How neighbor particles affect incipient particle motion in a laminar shear flow was captured by a high-speed camera in Agudo's studies (Agudo and Wierschem, 2012; Agudo et al., 2014). Initial locations of a moving particle, arrangements of neighboring particles, and geometries of the substrate have influence on incipient particle motion. Ten Cate et al. (2002) also used a high-speed camera to obtain the settling of a single sphere, and the time series of the particle settling velocity was provided by image processing. To address the fundamental mechanisms of solids suspension, our previous work (Mo et al., 2015) investigated the suspension characteristics of one spherical particle in a laminar flow generated by a rotating disk. The motions of a particle in the bottom and side views were provided by two cameras, and a matlab routine was programmed to track the centroid of the particle. It was derived that the suspending force is proportional to the impeller speed to the power 1.4 which is intermediate between viscous and inertial scaling. Direct numerical simulations based on the lattice-Boltzmann method (LBM) and resolved particle method (Derksen, 2012) were performed for the flow system, 
and the trajectory and vertical velocity of the particle was reproduced well with the critical conditions between simulation and experiment within a 3\% difference.

Our work as presented in the current article is an extension of the solid-liquid suspension for a single sphere. We increased the number of particles from one to eight and replaced the rotating disk with a standard Rushton turbine. The visualization experiments of multiple particles were presented, where the location of each particle could be distinguished and detected. The feasibility of aforementioned LBM simulation for multiple particles was verified through a detailed comparison with the experimental data. In this article, the first objective of research is to show visualization motion of a small number of particles during their lift-off process including trajectories of particles, suspension sequences, and possible patterns formed by particles before their suspension. The second objective is to verify the accuracy of the simulation based on the LBM for multiple particles by comparison with the experimental data, and then to investigate the effects of the flow field around the particles on their suspension behavior. The third objective is to further develop the relatively simple case described in this paper toward suspension mechanisms of more complex systems.

The paper is organized as follows: the experimental conditions including stirred system, flow conditions, and particle properties are given first. Next, the visualization experiments and image processing are introduced. In the subsequent section, the lattice-Boltzmann method, boundary conditions and lubrication force model are briefly described. In the results and discussion section, first the critical impeller speeds to lift off different numbers of particles are given. Then, the experimental suspension characteristics of multiple particles including lift-off sequences, trajectories, and velocities are discussed. In the third part, the simulated results are compared with the experimental data, and the role of the pressure field around particles is analyzed. The final section summarizes the conclusions and lists future research directions.

\section{Experiment setup}

\subsection{Flow system}


Geometric parameters of the stirred tank and the standard Rushton turbine are shown in Fig. 1. The main reason for choosing a square mixing tank is good optical access and no optical distortion by curved side walls. The side length of the cuboid tank is $T=0.22 \mathrm{~m}$. The diameter of the turbine is $D=0.11 \mathrm{~m}$, and the off-bottom clearance of the turbine is $C=0.25 T$. The liquid height inside the tank is $H=T$. As the flow is laminar (see the Reynolds number below), no waves develop and no air is entrained at the free surface so that it can be considered flat in experiments as well as in the simulations. The origin of the Cartesian coordinate system is located at the bottom center of the tank, as shown in Fig. 1.
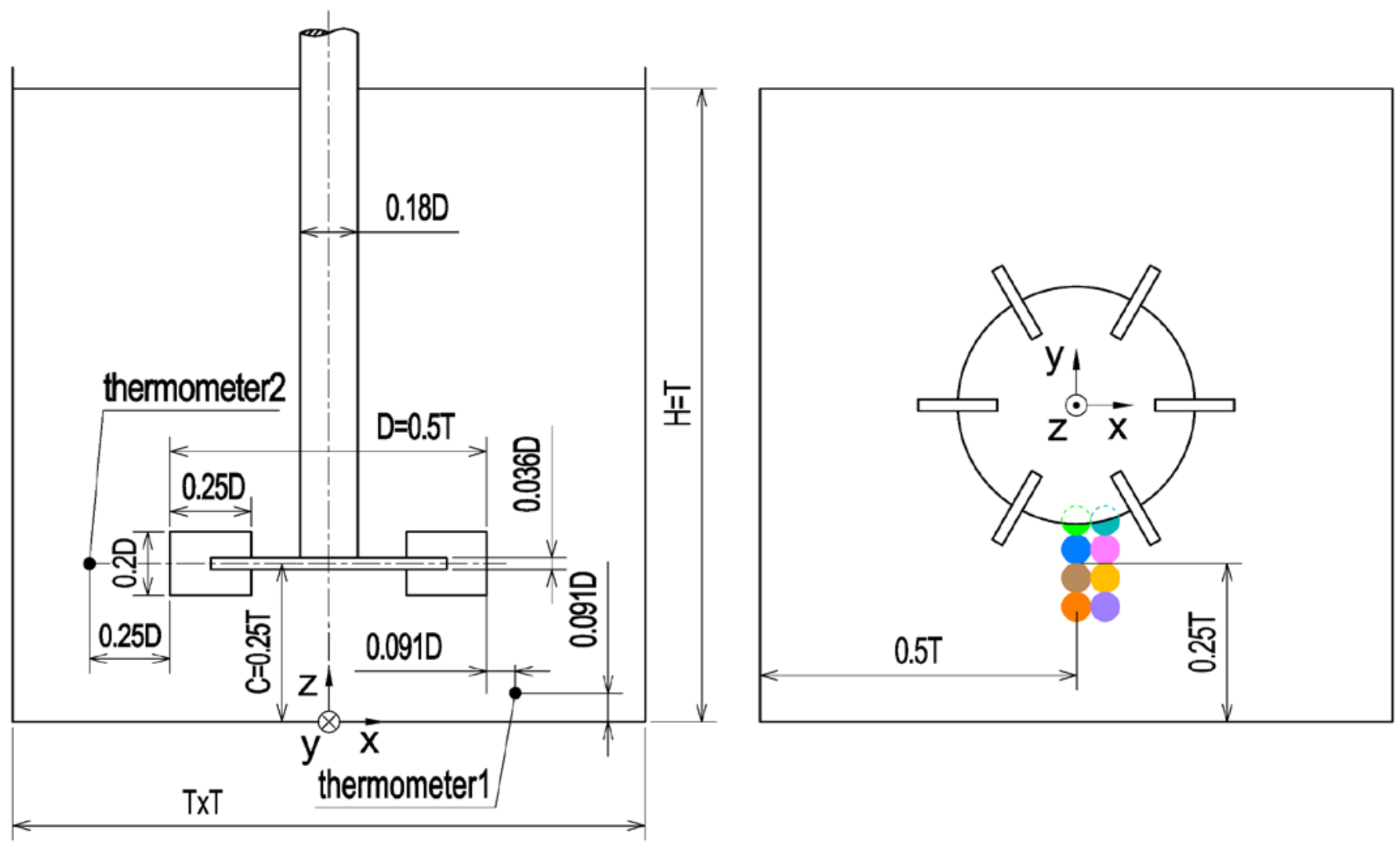

Fig. 1 - The geometry of the stirred tank agitated by a standard Rushton turbine (left panel, side view) and the initial positions of the eight particles (right panel, top view). The Cartesian coordinate system is located at the bottom center of the tank. The eight particles were painted different colors: red, brown, blue, green, purple, orange, pink, and cyan.

Dimethyl silicone oil (Shanghai Lubao Company, China) with density $\rho=978 \mathrm{~kg} / \mathrm{m}^{3}$ was chosen as working liquid. The viscosity of the silicone oil was measured by a MARS40 rheometer (Haake, Germany). The relationship between 
dynamic viscosity $\rho v$ (in Pa.s) and temperature $T_{\mathrm{m}}$ (in ${ }^{\circ} \mathrm{C}$ ) is $\rho v=3.1790-0.0438 T_{\mathrm{m}}$, where $v$ is the kinematic viscosity of the silicone oil (in $\mathrm{m}^{2} / \mathrm{s}$ ). Before and after each experiment, the temperature of the liquid was measured at two locations (see Fig. 1). The experimental average temperature was controlled at $20.2 \pm 0.1^{\circ} \mathrm{C}$, and thus the viscosity variation caused by the fluctuation of temperature is negligible.

Eight solid quartz spheres with diameter $d_{\mathrm{p}}=10 \pm 0.02 \mathrm{~mm}$ were selected. Eight was the maximum number of particles we could individually distinguish and detect in the current experimental setup. The density ratio between the particles and the working liquid is $\rho_{\mathrm{p}} / \rho=2.28$. In order to distinguish particles in experiments and detect their positions in post processing, we painted the particles with different colors. The initial locations of the particles in the experiments are also shown in Fig. 1.

The turbine was driven by an electric motor (ABB, Switzerland), and the rotational speeds were controlled by a 6SL3210 frequency converter (Siemens, Germany) and a shaft encoder (Kubler, Germany). During the experiments, the turbine was accelerated from rest to a target speed with a constant acceleration of 100 revolution/minute/s $(\mathrm{rpm} / \mathrm{s})$. For example, the target speed to lift off 8 particles is $230 \mathrm{rpm}$, so this speed is reached after $2.3 \mathrm{~s}$. The fluctuation of the target speed is less than $\pm 0.5 \mathrm{rpm}$. The Reynolds number based on impeller speed $N$ (in revolution/s) is defined as $\operatorname{Re}=N D^{2} / v$. The highest Reynolds number in our experiments is less than 20 so that the flow is laminar with, however, appreciable inertial effects. The ratio of inertial stress exerted on the particle to gravity-induced stress is represented as the inertial Shields number (Derksen, 2012) $\theta \equiv \rho N^{2} D^{2} / g \Delta \rho d_{p}$ with g gravitational acceleration (in $\mathrm{m} / \mathrm{s}^{2}$ ), and $\Delta \rho=\rho_{\mathrm{p}}-\rho$ density difference between solid and liquid (in $\mathrm{kg} / \mathrm{m}^{3}$ ).

\subsection{Visualization experiment and image processing}

One of the main purposes of this study is to quantify critical conditions for a number of particles getting suspended. As a metric, we defined the lowest impeller speed $N_{\text {LO }}$ for which particles could be lifted off the bottom of the tank within 1 minute. As we will see, $N_{\mathrm{LO}}$ depends on the number of lifted-off particles:

$N_{L O}=f\left(n_{L O}\right), n_{L O}=1 \mathrm{~L} 8$. The 1 minute restriction was mainly determined to save computational resources in our simulations. For example, if the lift-off process at $N_{\mathrm{LO}}=230 \mathrm{rpm}$ lasts 1 minute in an experiment, we need to simulate this process with at least 230 impeller revolutions. A longer lift-off process means that more impeller revolutions need to be simulated which is very time-consuming. The uncertainty of the $N_{\text {Lo }}$ measurement is less than 
$2 \%$; that is, if we decrease the $N_{\mathrm{LO}}$ by $2 \%$, the particles cannot be lifted off. Each experiment has been performed at least two times to confirm the experimental $N_{\mathrm{LO}}$ results.

As shown in Fig. 1, the initial locations of the eight particles are away from the bottom center of the tank. The first stage of the particles' movements is their rolling motion towards the bottom center. Then, multi-particle interactions happen near the bottom center in the second stage. In the third stage, the particles will be gradually lifted off and will rise towards the turbine. The particles' movements in these three stages are discussed in detail in this paper. The interactions between particles and the impeller, especially its blades, are quite random, and not considered in this study.

After the measurement of $N_{\mathrm{LO}}$, the multi-particle motions were captured by using two high-speed cameras (Dantec Dynamics A/S, Denmark), as shown in Fig. 2. The particles rolling over the bottom wall were captured by the camera A looking through bottom, and the particles being lifted off the bottom wall by the camera B looking through the side wall. Two strong lights were used to obtain clear and distinct particle images. The measurement area and the velocities of particles both dictate the resolution and the capture frequency of the camera. The motion of particles rolling on the bottom wall was slow, and then a high resolution of $1280 \times 720$ pixel $^{2}$ and a low capture frequency of 60 frames per second (fps) were used to capture the multi-particle trajectory. During the lift-off stage, the velocities of multi-particle system were high, and thus a slightly lower resolution of $1000 \times 1000$ pixel $^{2}$ and a much higher frequency of $300 \mathrm{fps}$ were necessary.

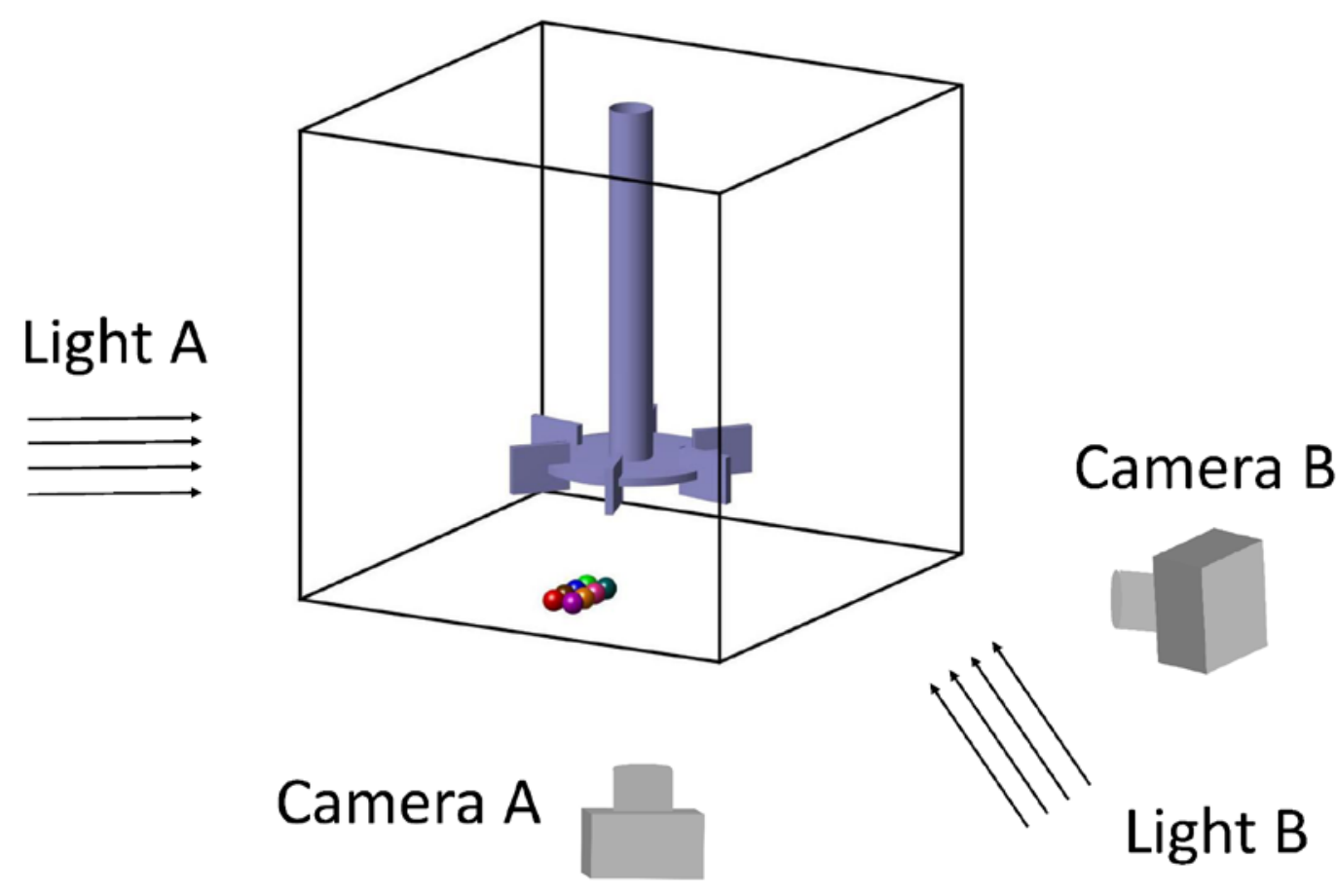


Fig. 2 - Sketch of image capture in the experiments: Camera A captures the bottom view; Camera B the side view.

The raw images obtained by the two cameras were processed and analyzed by a code in the matlab environment (version: R2017, MathWorks, USA) to determine the centroids of each particle as a function of time. In this study, the matlab function imfindcircles (the MathWorks Inc., 2017; Woo et al., 2016) based on a Circular Hough Transform (CHT) (Davies, 2005) algorithm was used to find all the circles in the images (Atherton and Kerbyson, 1999). The analysis process required a number of input parameters: the range of pixels over the radius of a particle was given first. The Object polarity was set to 'Bright' due to the particles with color in raw images. Furthermore, the Sensitivity factor was set to 0.97 or 0.98 to decrease invalid detected circles, and the Edge gradient threshold to 0.01 to increase the contrast on the circular edge of spheres in images. In addition, the Central Difference with fourth-order accuracy (Dilloo and Tangman, 2017) was used to weaken the fluctuation of detected centroids on calculating the lift-off velocity of particles. Typical image samples captured from the bottom view and from side view are shown in Fig. 3. Detection for particle motion on the bottom wall is automatic. Sometimes, there are some interruptions while detecting the lift-off of particles because the particle we are interested in might be hidden behind other particles during lift-off. In such cases, a manual operation to connect the interrupted particle trajectories is necessary.
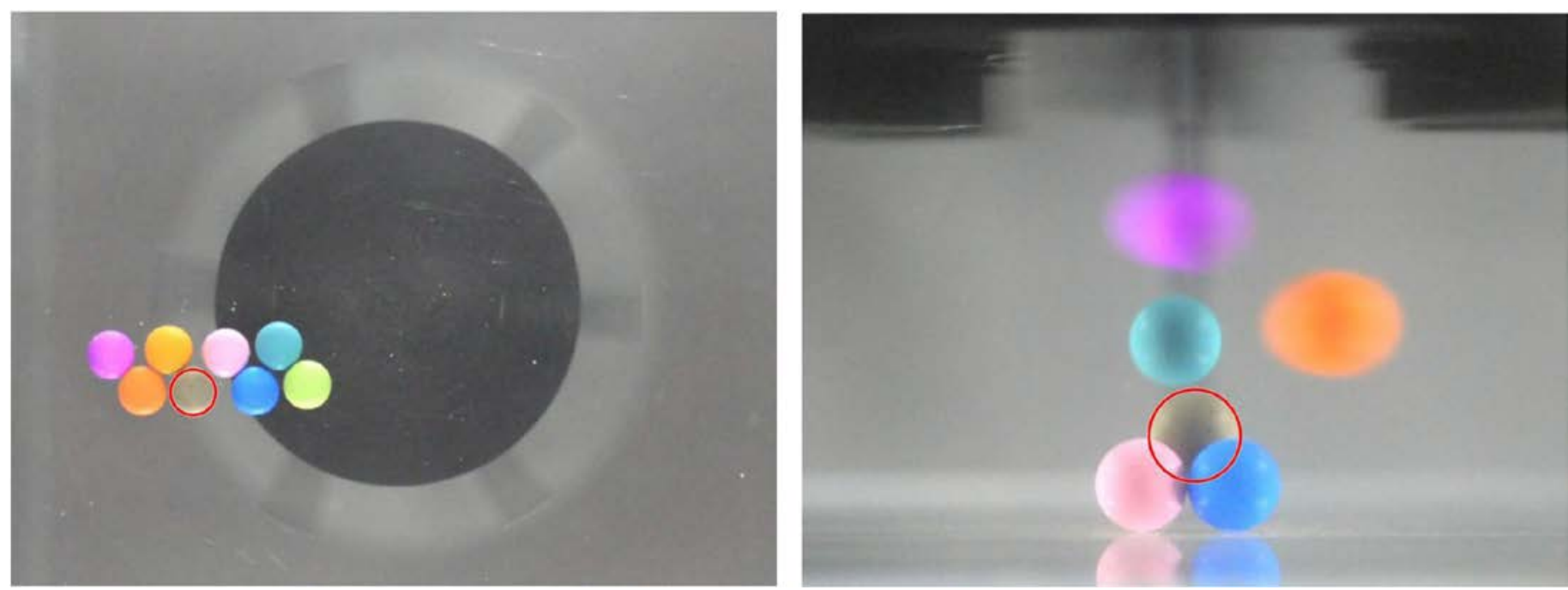

Fig. 3 - Detection of particle position. The brown particle is shown as an example. Left panel: particles roll on the bottom wall of the tank. Right panel: the brown particle is lifted off. 


\section{Numerical approach}

The lattice-Boltzmann method (LBM) (Chen and Doolen, 2012; Succi, 2001) was used to solve the liquid flow fields in this article. Details about the specific LBM scheme used are given by Somers and his coworker (Eggels and Somers, 1995; Somers, 1993). In this method, space is discretized in a uniform, cubic grid. The scales of space and time are defined as the "lattice units"; that is, the grid spacing $\Delta$ and time step $\Delta t$ are unit length and time respectively. The physical parameters of the experiments were translated to dimensionless numbers in the simulations. For the moderate, sphere-based Reynolds numbers, a resolution of 12 grid spacings over a sphere diameter was sufficient to resolve the fluid flow around a particle (Derksen, 2012). For a $10 \mathrm{~mm}$ sphere, therefore, the overall grid in the cubic tank consisted of $264^{3}$ cells, and the diameter of the Rushton turbine was represented by 132 $\Delta$. The governing equations derived from the basic model in LBM are formally equivalent to the Navier-Stokes equations for compressible fluids. Then, the speed of the fluid $|\mathbf{u}|$ (in lattice units) must be much lower than the speed of sound $c_{s}=\sqrt{2} / 2$ (Ten Cate et al., 2002) to assure incompressible flow. If the tip speed of impeller $\left(v_{\text {tip }}=\pi N D\right)$ is less than 0.1 lattice units, the above criterion will be achieved $(\mathbf{u g l}=1)$. In terms of computer time, this restriction implies that one impeller revolution needs a large number of time steps (of the order of a few thousand).

A halfway bounce-back boundary was used to the bottom and the side wall of tank to implement their no-slip condition (Ziegler, 1993). The free-slip liquid boundary at the top of tank was imposed by a halfway specular reflection rule (Yamamoto et al., 2001). An adaptive force-field technique (or immersed boundary method) (Derksen and Van den Akker, 1999) was imposed for non-square geometries and moving objects, such as the six blades of the impeller, the shaft of the impeller, and the particle surfaces. Taking a moving sphere as example, the particle surfaces are defined as sets of off-grid, closely spaced points. The spacing between points is less than one lattice unit, and its typical value is $0.7 \Delta$. If there is a velocity difference between surface points of a particle and 
interpolated points of fluid, a local force is applied on the fluid. According to an iterative algorithm involving the force (with two relaxation factors $\boldsymbol{\alpha}$ and $\boldsymbol{\beta}$ ), the velocity difference is decreased and no-slip is approached (Ten Cate et al., 2002). Integrating the local forces to achieve no slip over the particle surface provides the hydrodynamic force and torque on the particle. In addition, the net gravity and the subgrid lubrication force on the sphere are also used to update the particle's translational and rotational motion. These results determine the new locations, translational and rotational velocities of the sphere surface points. This establishes two-way coupling between fluid and particle.

A hard-sphere model with two parameters (a restitution coefficient $e$ and a friction coefficient $\mu$ ) was applied to deal with the collisions of a sphere with the container wall and mutual collisions of spheres (Mo et al., 2015). In a solid-liquid flow, the loss of momentum upon contact is negligible compared to the dissipation in the liquid upon the approach of two particles. Therefore it is appropriate to set $e=1$ in the simulations. A study on the granular bed erosion suggests that the friction coefficient $\mu$ determines the particle motion of rolling over the surface of the particle bed (Derksen, 2011). In the subsequent discussion, we explore the influence of friction coefficient on multiple-particle suspension. The collision between particles and Rushton turbine is akin to soft spheres interactions (Derksen, 2012): once the overlap of any particle with the impeller is over $0.5 \%$ of the particle volume, a repulsive force is applied; the order of magnitude of collision time is $10 \Delta t$, which corresponds approximately to $1^{\circ}$ of impeller rotation.

Since the particles move over a fixed grid, the flow in between particles cannot be resolved when two particle surfaces are very close. By introducing subgrid lubrication forces, we compensate for this effect (Nguyen and Ladd, 2002). The lubrication force model involves two parameters in order to correctly reproduce physical situations. One is the distance between two solid surfaces $s_{0}$ below which the lubrication force switches on. The other is the threshold $s_{1}$ below which the lubrication force saturates. Assuming creeping flow in between the solid surfaces (Kim and Karrila, 2005), the expression for the lubrication force reads: 
$F_{\text {lub }}=6 \pi \rho v \frac{a_{i}^{2} a_{j}^{2}}{\left(a_{i}+a_{j}\right)^{2}}\left(\frac{1}{s_{1}}-\frac{1}{s_{0}}\right)\left(\mathbf{n} \cdot \Delta \mathbf{u}_{i j}\right) \quad s \leq s_{1}$

$F_{\text {lub }}=6 \pi \rho v \frac{a_{i}^{2} a_{j}^{2}}{\left(a_{i}+a_{j}\right)^{2}}\left(\frac{1}{s}-\frac{1}{s_{0}}\right)\left(\mathbf{n} \cdot \Delta \mathbf{u}_{i j}\right) \quad s_{1}<s<s_{0}$

$F_{\text {lub }}=\mathbf{0}$ $s \geq s_{0}$

$F_{\text {lub }, \mathrm{i}}=F_{\text {lub }} \mathbf{n}, \quad F_{\text {lub }, \mathrm{j}}=-F_{\text {lub }} \mathbf{n}$

where $\mathbf{n}$ is the unit normal vector from the center of particle with radius $a_{\mathrm{i}}$ to the other one with $a_{\mathrm{j}}$, and the relative velocity $\Delta \mathbf{u}_{i j}$ represents $\mathbf{u}_{\mathrm{pj}}-\mathbf{u}_{\mathrm{pi}}$. In this study, $s_{0}=0.1 d_{\mathrm{p}}, s_{1}=10^{-4} d_{\mathrm{p}}$.

\section{Results and discussion}

\subsection{Critical impeller speed to lift off particles $N_{\mathrm{LO}}$}

Fig. 4 shows the critical impeller speed to lift off particles $N_{\text {LO }}$ as a function of the number of suspended particles $n_{\llcorner O}$. It is as expected that $N_{\llcorner O}$ increases with the increase of $n_{\llcorner O}$ because it requires more energy to suspend more particles. In the investigated eight-particle system, we did not encounter a situation where six or seven particles were being lifted off; $N_{\mathrm{LO}}=214 \mathrm{rpm}$ at $n_{\mathrm{LO}}=5$, and $N_{\mathrm{LO}}=230 \mathrm{rpm}$ at $n_{\mathrm{LO}}=8$. We tested three impeller speeds between 214 and $230 \mathrm{rpm}$, namely 218, 222, and $226 \mathrm{rpm}$, and only five particles could be lifted off. Then we increased the impeller speed to $230 \mathrm{rpm}$, and the three remaining particles were lifted off.

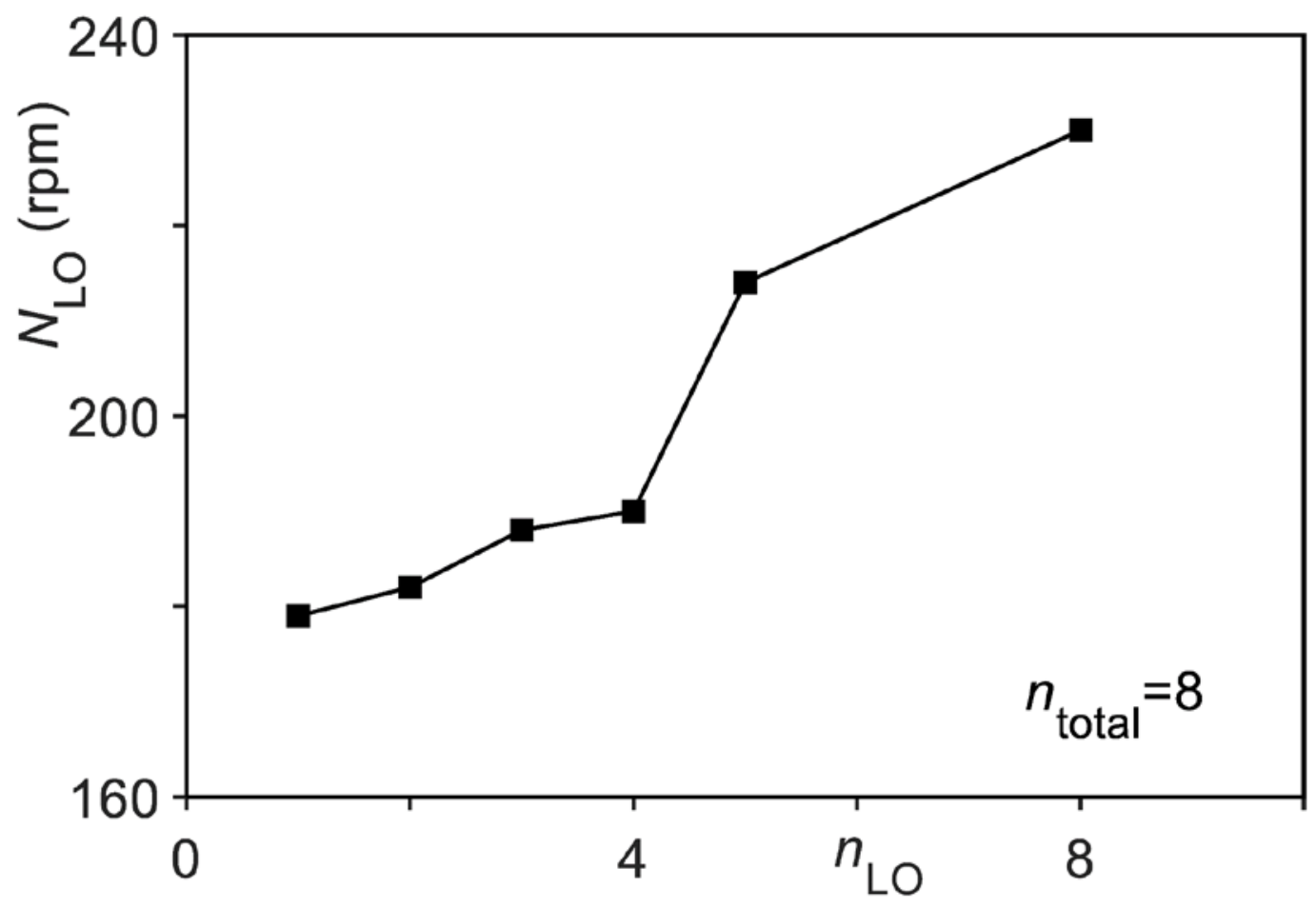


Fig. 4 - Critical impeller speed to lift off particles $N_{\mathrm{LO}}$ versus suspended particle number $n_{\mathrm{LO}} . n_{\text {total }}=8$ means the total particle number is 8 . In the experiments, we did not observe the phenomena that 6 or 7 particles could be lifted off $\left(n_{\llcorner O}=6\right.$ or 7$)$.

The apparent increases of the impeller speeds required for lifting off 5 particles $\left(n_{\llcorner O}=5\right)$ and 8 particles are caused by the formation of two stable particle patterns on the bottom of the tank, as shown in Fig. 5. They consist of four and three particles respectively and require a strong flow (and thus a high impeller speed) to be broken up. When four particles are rolling on the bottom, a rhombus pattern is formed. An increase of about $13 \%$ in terms of impeller speed is needed to suspend the fifth particle (i.e. destroy the rhombus pattern) in comparison with the critical impeller speed $N_{\mathrm{LO}}=190 \mathrm{rpm}$ at $n_{\mathrm{LO}}=4$. To destroy the triangle pattern, an additional $7 \%$ increase in impeller speed is required, as compared with the $N_{\mathrm{LO}}=214 \mathrm{rpm}$ at $n_{\mathrm{LO}}=5$. It is interesting that once the triangle pattern is broken at $N_{\mathrm{LO}}=230 \mathrm{rpm}$, all the three particles will be subsequently lifted off at that impeller speed. We measured the critical lift-off speed in an experiment with only one particle and found that the speed was $218 \mathrm{rpm}$, which further confirms the influence of the stable triangle particle patterns and could explain the subsequent lift-off of the three particles. In industrial processes, the final suspension of very small amounts of solids usually requires a large increase of impeller speed, and thus a significant increase of mechanical energy input (Tamburini et al., 2012). Stable particle patterns might be a reason for this.
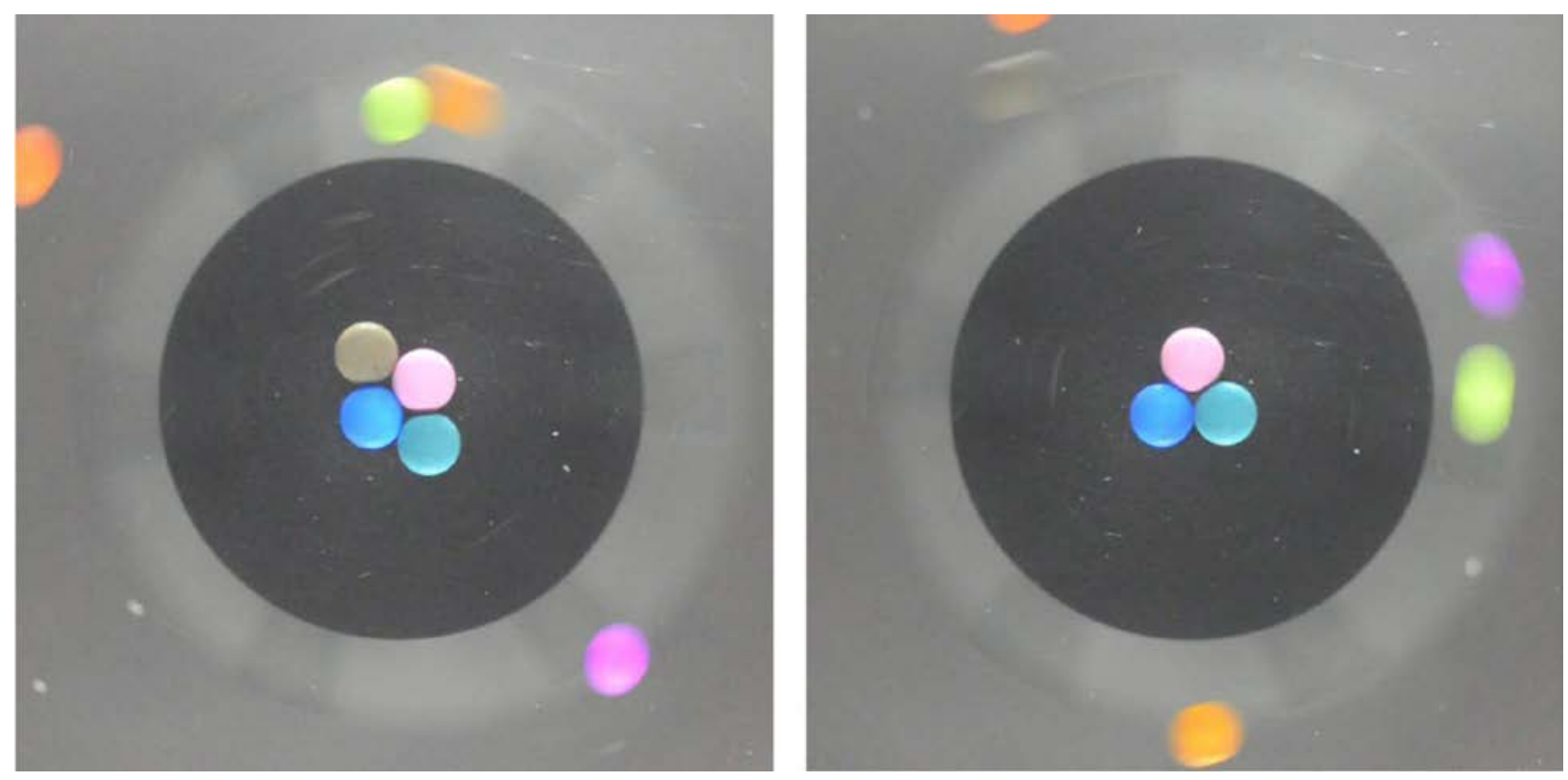
Fig. 5 - Two stable patterns formed by particles rolling near the center of the bottom wall. Left panel: rhombus pattern with four particles; right panel: triangle pattern with three particles.

\subsection{Experimental characteristics of multi-particle suspension}

The first three stages of particle motion, namely rolling over the bottom, interacting and being lifted off around the bottom center, and rising along the center line of the tank, are our particular interests in the visualization experiments. The complete suspension of the eight particles will be discussed as a typical case in the following subsections. In the experiment, the impeller was accelerated from rest to $N_{\mathrm{LO}}=230 \mathrm{rpm}$ with a constant acceleration of $100 \mathrm{rpm} / \mathrm{s}$; that is, the required time for achieving $N_{\mathrm{LO}}$ is $2.3 \mathrm{~s}$.

Fig. 6 shows the trajectories of the particles rolling from the initial positions to the bottom center in two realizations of the experiment. The full particle trajectories in realization R1 are plotted to give an impression of the particle motion over the bottom. For the particles being suspended later, such as the blue and cyan particles, they move around the bottom center for a long time, resulting in partly overlapping trajectories. During this stage, the unsuspended particles strongly interact with each other, and their trajectories are to some extent random (e.g. due to surface roughness of the particles and the tank bottom). For clarity, we show in Fig. 6 the trajectories of the second realization (R2) only when the particles are outside a circle with diameter $4.0 d_{p}$ around the center of the tank bottom. In that sense, the two realizations of the experiment give highly reproducible results. In the two repetitions of the experiment, the purple particle was lifted off first when it was outside the circle with diameter $4.0 d_{p}$. The time from the moment of the impeller starting to the moment the purple particle was lifted off over a vertical distance of $0.1 d_{p}$ was $6.5 s$ in realization $R 1$, and it was $6.3 s$ in R2. This is longer than the impeller acceleration time of $2.3 \mathrm{~s}$. The Reynolds number is 19.0 in this experiment. Our previous research reported that the laminar flow reached steady state very shortly after the end of the accelerating process of the impeller (Mo et al., 2015). Thus, at the moment that the purple particle was lifted off, the overall flow field in the tank had already reached steady state. The distance of momentum diffusion could be estimated as $\sqrt{v t}$ with $v=0.00235 \mathrm{~m}^{2} / \mathrm{s}$ the kinematic viscosity of the silicone oil. Over $t=1 \mathrm{~s}, \sqrt{v t} \approx 48 \mathrm{~mm}$; the flow development in the tank thus only lasted a few seconds. 


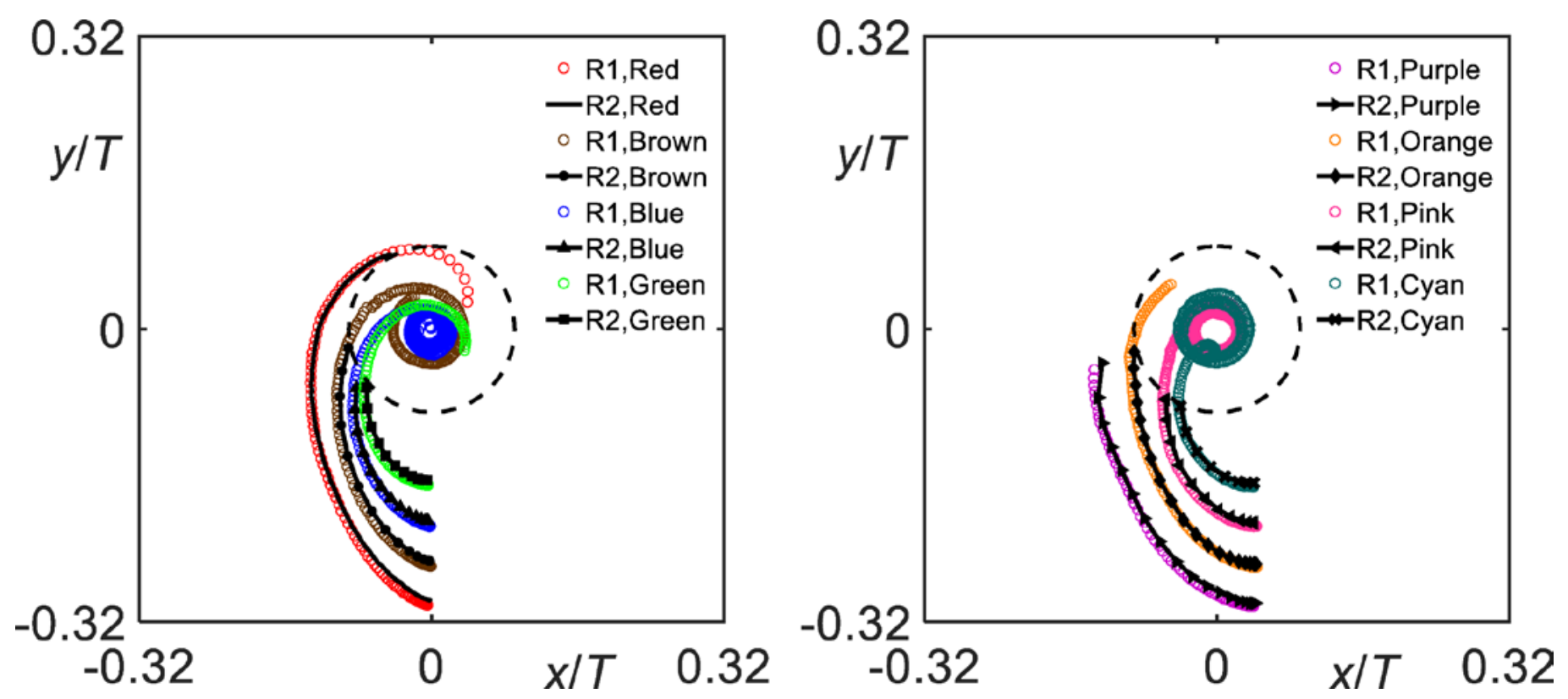

Fig. 6 - Trajectories of the eight particles rolling on the bottom of the tank. R1 and R2 denote two realizations of the experiment. Particles in different initial positions are represented by different colors in the panels. The full trajectories of the particles in R1 are shown. For clarity, the particle trajectories of R2 are not shown when the particles move into the dashed circle with diameter of $4.0 d_{p}$ around the center of the bottom.

While the particles approach the center of the bottom, they are gradually lifted off. Three realizations of the experiment are performed, and the particle lift-off sequence and particle pattern in two realizations are shown in Fig. 7. Although the flow field is laminar, the particle lift-off sequence is to some extent random in different realizations of the experiment. The time from the moment of the impeller starting to the moment each particle was lifted off over a vertical distance of $0.1 d_{p}$ is random as well. During the lift-off process of a multi-particle system, one particle will interact with other particles, climb and roll over them, and then be lifted off. We consider that the precise contact position and the friction coefficient at the contact point (i.e. the local surface roughness) are the main reasons for the random lift-off sequence. 

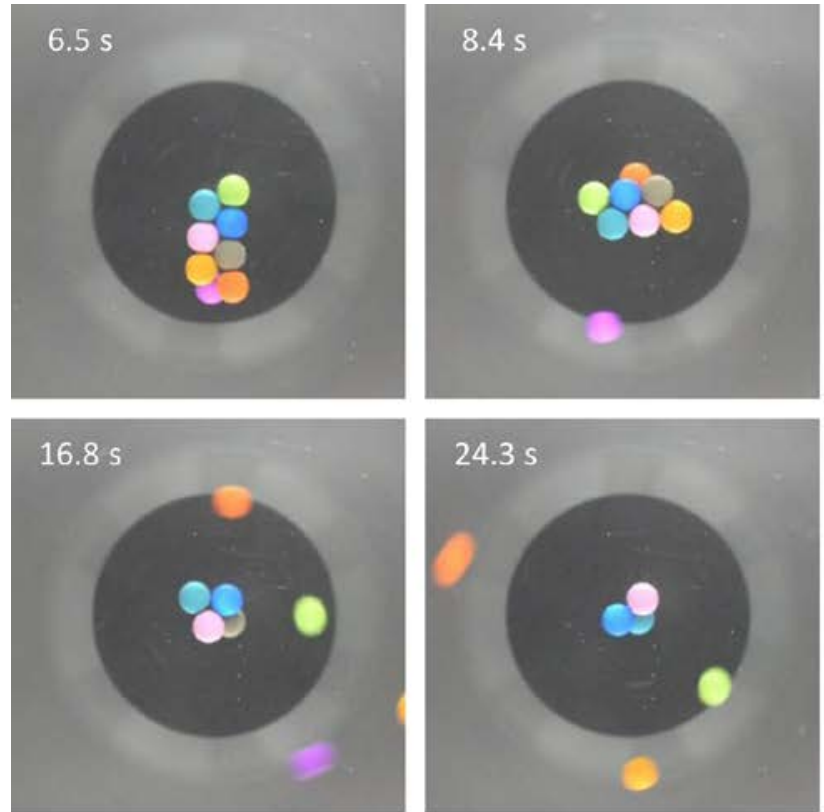

(a)
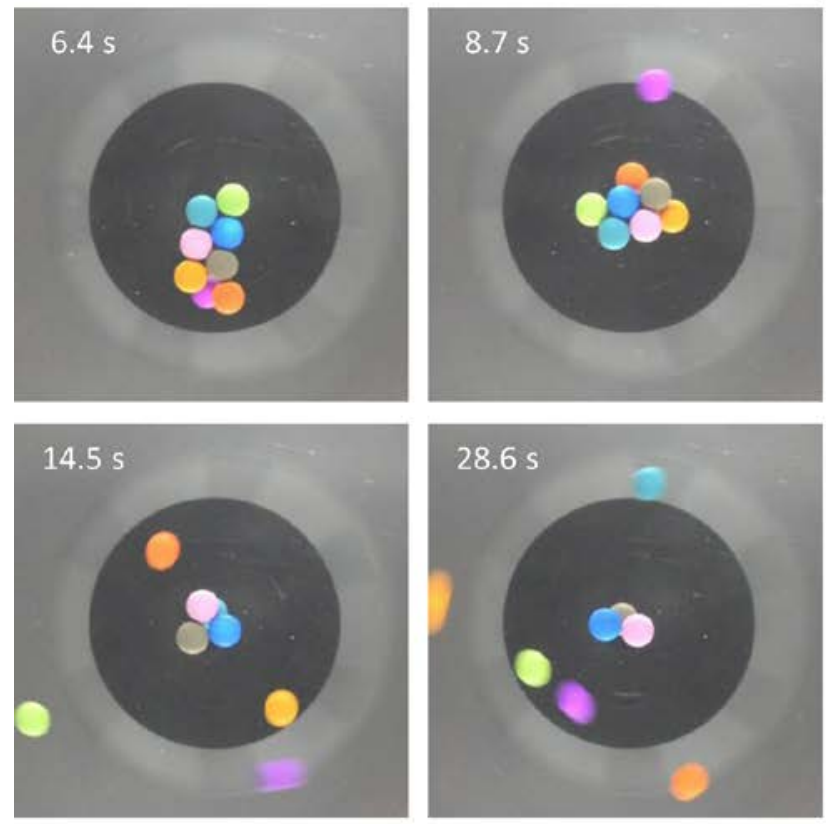
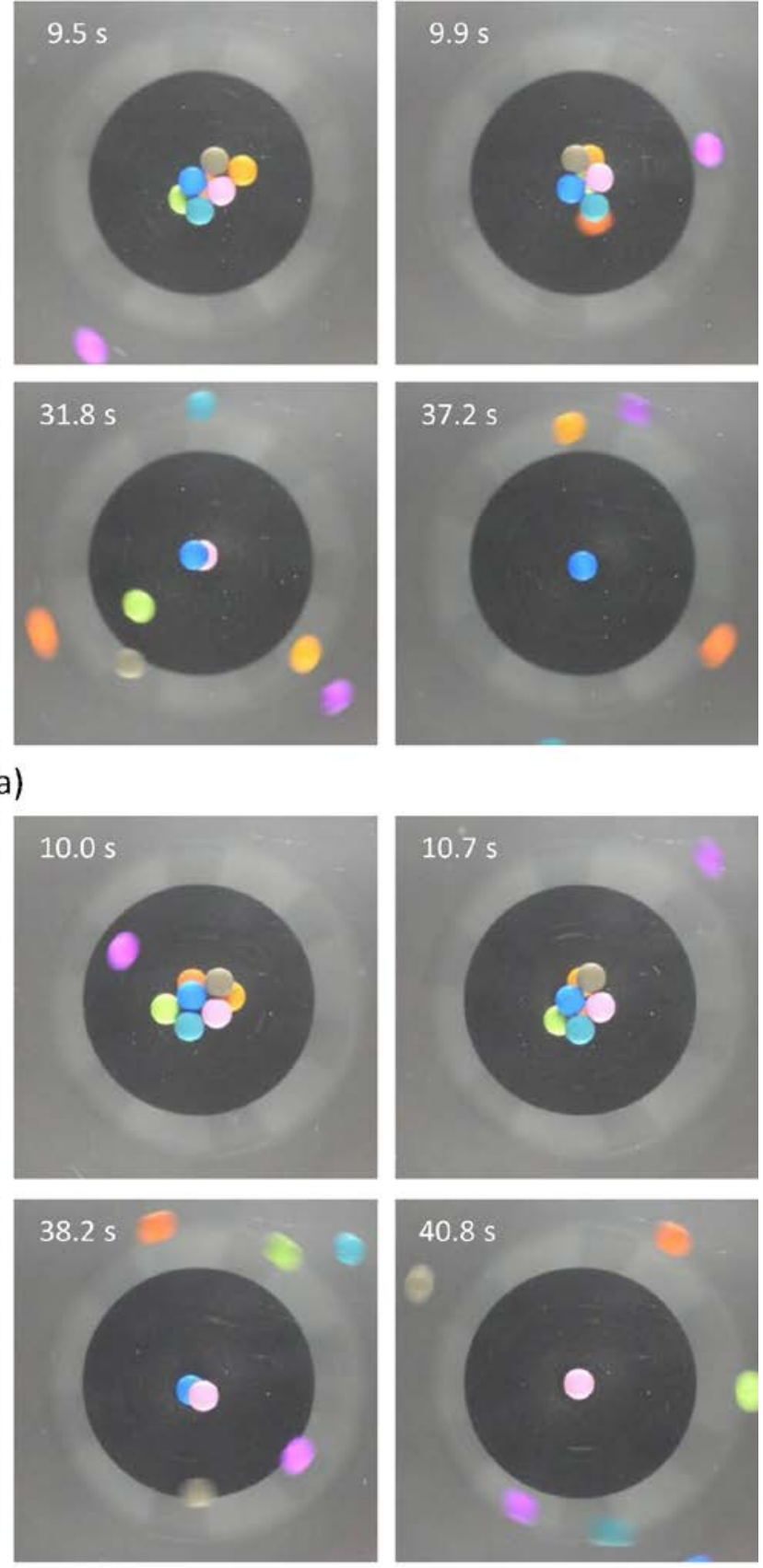

(b)

Fig. 7 - The particle lift-off sequences and particle patterns in two realizations of the experiment. Particles were viewed from the bottom wall of the tank. The lift-off sequence of R1 in panel (a) is purple, red, green, orange, brown, cyan, pink, and blue; the sequence of R3 in panel (b) is purple, red, orange, green, cyan, brown, blue, and pink. The time from the moment of the impeller starting to the moment each particle was lifted off over a vertical distance of $0.1 d_{\mathrm{p}}$ was given in each panel.

Fig. 8 shows the time series of particles trajectories and velocities during lift-off. The origin of the time axis $(t=0)$ in the figure is set at the moment that the distance from the center of the particle to the bottom (z) is equal to $1.0 d_{p}$. 
The vertical velocities of each particle were calculated from the vertical particle position as a function of time by a 4th order central difference formula; this differentiation reduced the effect of noise in the position data on the resulting velocity. At the moment that $z=1.0 d_{p}$, the particle being lifted off is climbing up other particles, and random contact among particles and associated random surface roughness exist. These random characteristics affect the particle trajectories and velocities in the initial lift-off stage. The results of the purple particle in two realizations of the experiments differ slightly, as well as those of the cyan particle. However, the maximum velocities of all particles are well reproducible. In terms of the vertical location of particles, the increase of $z$ is slow shortly after $t=0$; that is, the slope of the curve $z / C$ is small, except for the last suspended particle (the blue one), as shown in Fig. 8a. The changes in particles vertical locations result in fluctuations of particle vertical velocities; except for the last particle, all other particles experience a vertical velocity fluctuation shortly after $t=0$. These fluctuations mean that particles climb up other particles during their lift-off. For the last particle that is lifted off, its velocity increases without disturbance from other particles, and its maximum velocity is larger than the ones of the earlier suspended particle. During the lift-off of the cyan particle in realization 2, another particle that had been lifted off moved in front of the cyan particle; the boundary of the cyan particle in the captured images was partly hidden. As a result, a discontinuous particle trajectory and oscillating velocity appear in Figure 8. 

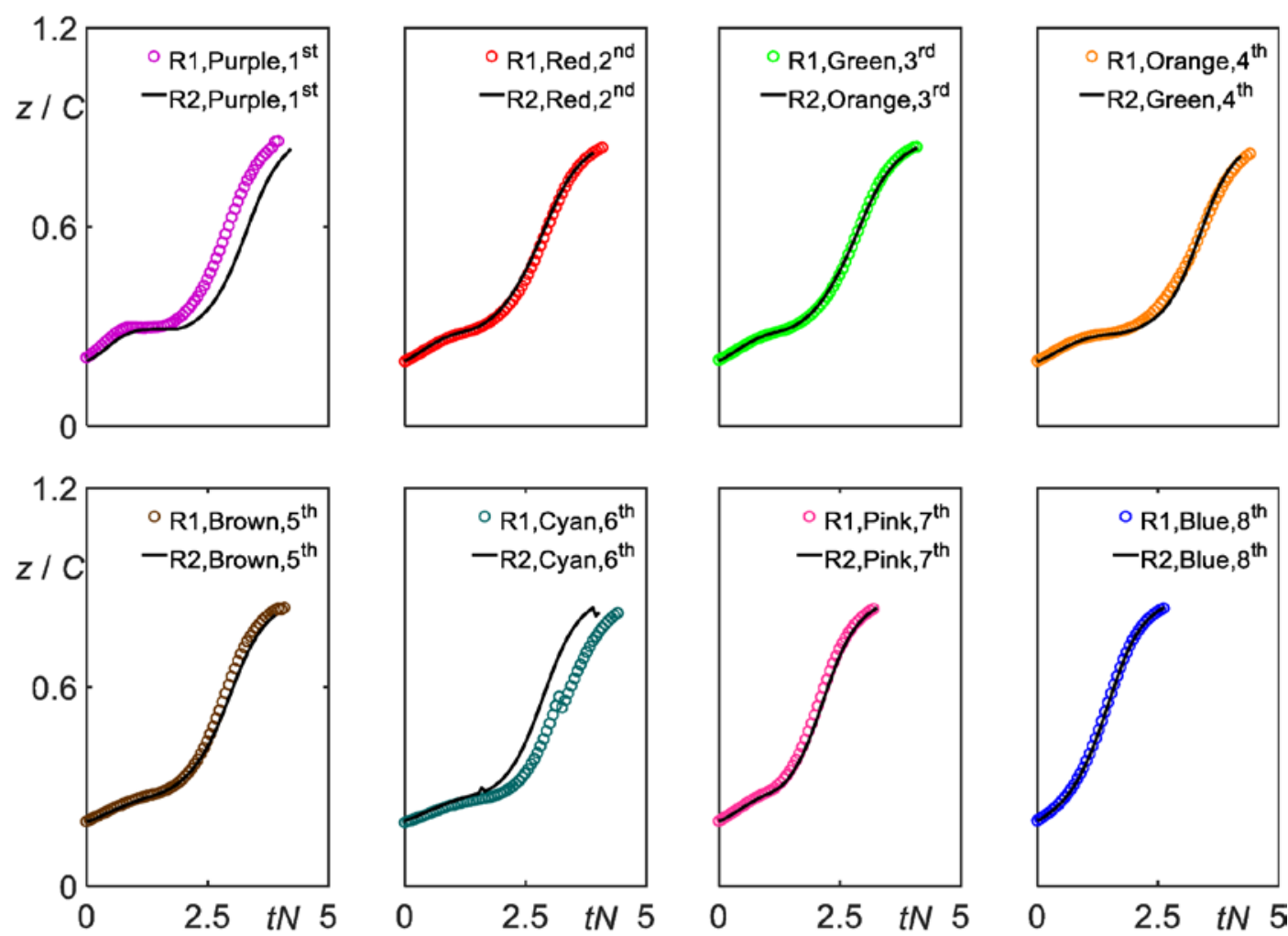

(a)
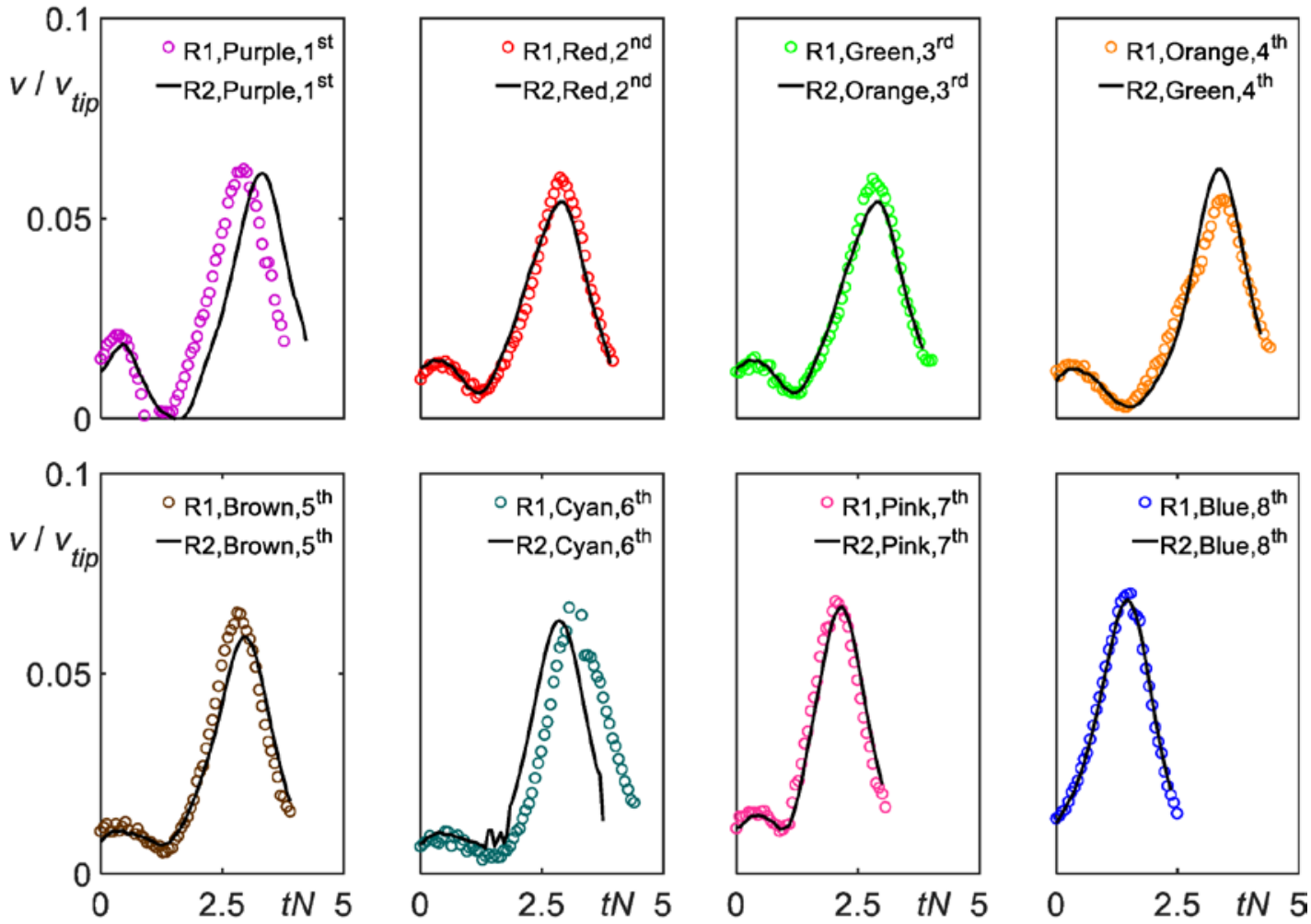

(b)

Fig. 8 - Time series of particle trajectories (a) and velocities (b) during particle lift-off along the centerline of the tank. The start times $t=0$ in the panels is set at $z=1.0 d_{p}$ with $\mathrm{z}$ the off-bottom displacement of particle center. R1 and R2 denote two realizations of the experiment. Different colors represent particles in different initial positions. The last number in each legend is the particle lift-off sequence. 
In the next subsection, the experimental results will be confronted with numerical simulations. The simulations resolve the liquid flow generated by the Rushton turbine. This information is not available from the existing visualization experiment. The data as measured in experiments provide important references to verify the accuracy of simulations we used. In return, the simulated results of interaction between the particles and the liquid could be used to explain some of the experimental phenomena.

\subsection{Simulated flow field and multi-particle lift-off}

The complete suspension of eight particles was simulated under the same conditions as used in the experiment. The data summarized in Table 1 reflect the situation after 200 impeller revolutions ( $t \approx 60 \mathrm{~s}$ in real time). As can be seen in Table 1, in the simulations only 4 particles get lifted off if $\Theta=1.382$ (which corresponds to $N=226 \mathrm{rpm}$ ). As also noted in our previous study (Mo et al., 2015), lifting off particles requires a slightly higher Shields number in simulations than in experiments. For that reason we performed simulations at $\Theta=1.520$ (i.e. $10 \%$ higher than 1.382; $10 \%$ in Shields number corresponds to $5 \%$ in impeller speed). In that situation, and under standard simulation conditions (a friction coefficient of 0.1 and no application of the lubrication force), all 8 particles get lifted off (see Table 1).

Table 1 - Simulated results of the eight particles system with Reynolds number Re=19.0 after 200 impeller revolutions (about one minute). $n_{\llcorner O}$ means the number of the particles that could be lifted off at a Shields number $\Theta$.

\begin{tabular}{ccccc}
\hline Case & $\Theta$ & $n_{\mathrm{LO}}{ }^{*}$ & Friction coefficient & with $F_{\text {lub }}$ \\
\hline S1 & 1.382 & 4 & 0.1 & no \\
S2 & 1.520 & 8 & 0.1 & no \\
S3 & 1.520 & 5 & 0 & no \\
S4 & 1.520 & 5 & 0.25 & no \\
S5 & 1.520 & 4 & 0.1 & yes \\
\hline
\end{tabular}


The friction coefficient $\mu$ significantly impacts on the number of suspended particles. By referring to published data (Derksen, 2011), we attempted three values of $\mu(0,0.1$, and 0.25$)$ in the simulations. No friction ( $\mu=0$, case S3) leads to unphysical behavior; that is, no particle rolls over the bottom wall. Larger friction between particles (as in case S4) also hinders the lift-off process. Therefore, the friction coefficient of 0.1 might be a good choice for simulating the suspension process. The introduction of the lubrication force model did not affect the critical suspension of particles; it did, however, affect the waiting time before particle suspension (Mo et al., 2015). For the case S5, we believe all the particles could be lifted off the bottom wall if the number of simulated impeller revolutions is increased; this, however, would be beyond the time limit in the experiment (1 minute). The simulated case $\mathrm{S} 2$ will be used in the following discussion.

Fig. 9 gives impressions of the flow in the mixing tank - in the presence of 8 particles - at $t N=30$. The vertical cross-sections represent the $x-z$ plane through the center of the tank; the horizontal cross-section is at $z \approx 5.4 \mathrm{~mm}$ which is the approximate vertical location of the center of a particle in contact with the bottom. As the number of particles is small, there is no apparent influence of the particles on the overall flow field, except for the direct vicinity of the particles. Therefore, the overall non-axially symmetric flow field in the right panel of Figure 9 is caused by the square base of the tank.

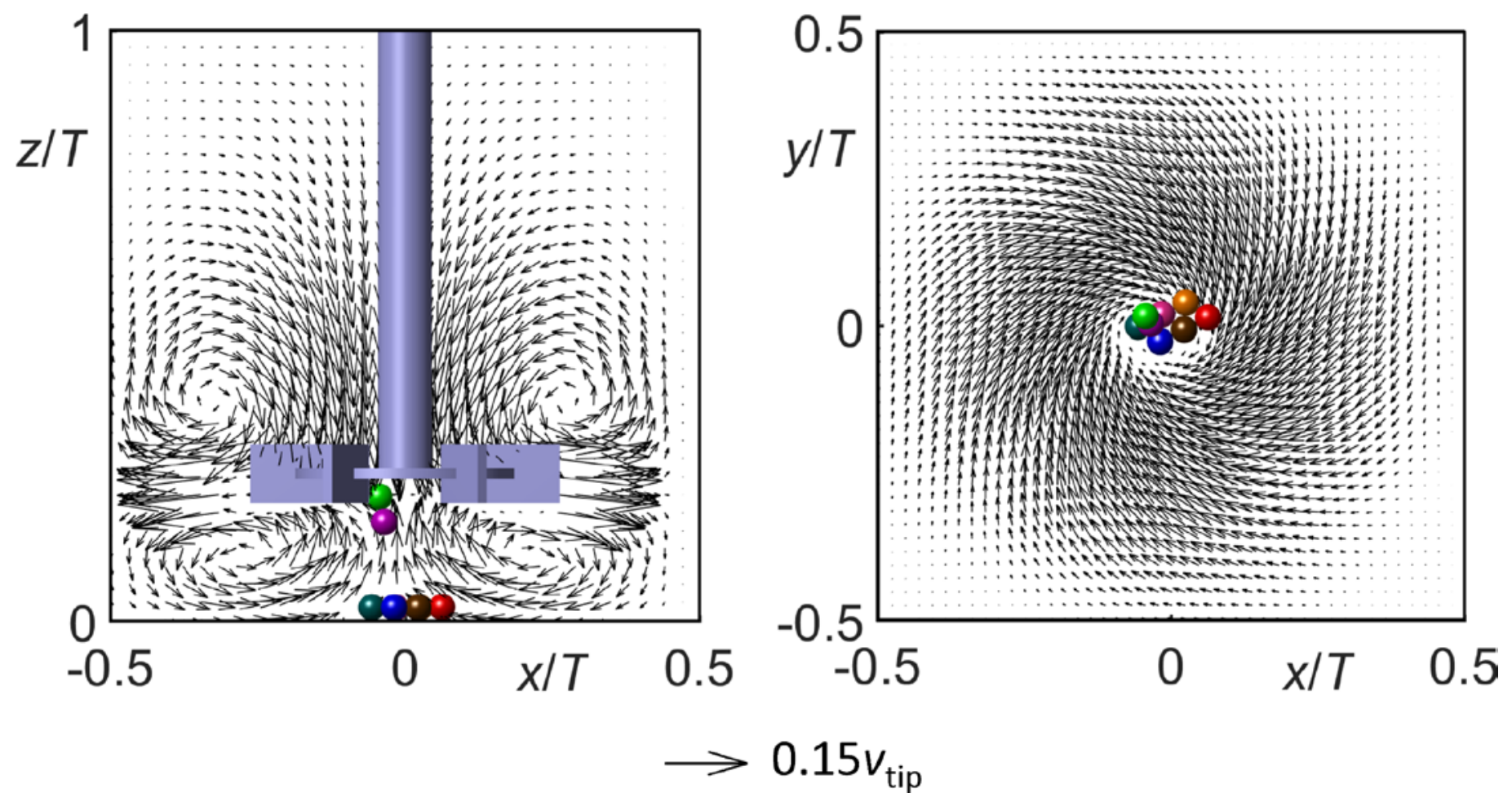


Fig. 9 - Instantaneous flow field after 30 impeller revolutions in a vertical cross-section ( $x-z$ plane, left panel) and in a

horizontal cross-section at $z \approx 5.4 \mathrm{~mm}$ (which is the approximate vertical location of the particle center, right panel).

The multi-particle motion as simulated will be compared with the experimental data in the following. In terms of the lift-off sequence, the results in the simulation are different to those observed in the experiment (see Fig. 7 and Fig. 10). In the experiment, the lift-off of the purple particle happened in the early stage of the bottom motion, and the first two particles getting suspended were from the same side. The simulated lift-off, however, only happened once the particles clustered and interacted around the center of the bottom of the tank, as shown in Fig. 10. In the simulation, the first particle was lifted off from one side of the particles cluster, but the second particle from the opposite side. So did for the third and fourth particles. The simulated trajectory of the purple particle (see Fig. 11) further confirms that the simulated particle suspension is slower than that in the experiment. In the simulation, the purple particle was lifted off within the dashed circle of diameter $4.0 d_{p}$, while it was lifted off outside the circle in the experiment. Except for this particle, the simulated trajectories of other particles are in very good agreement with the experimental data outside the circle, as shown in Fig. 11. The comparison for data inside the circle is omitted because of the randomness we mentioned before.
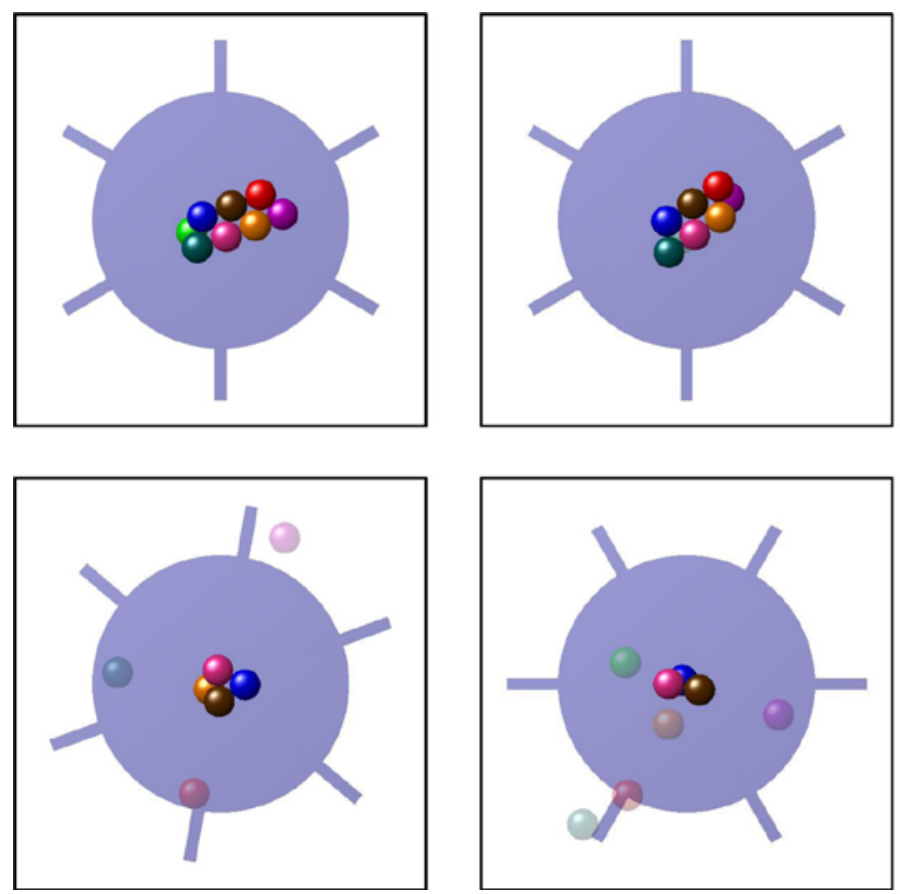
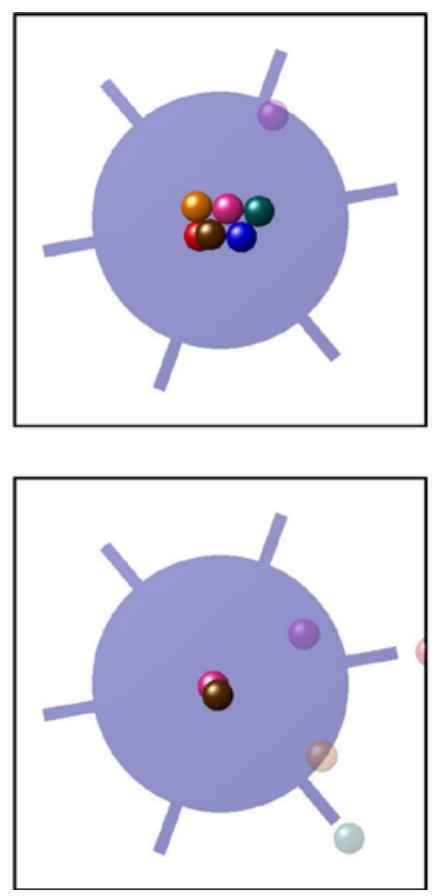
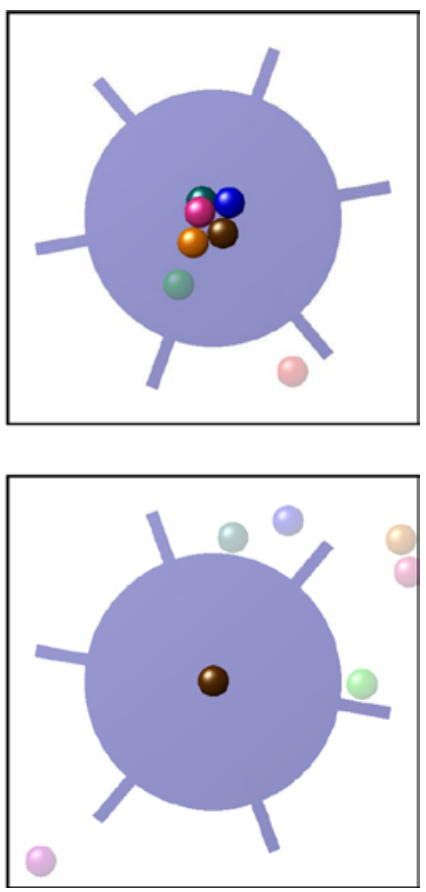
Fig. 10 - The simulated particle lift-off sequence (green, purple, red, cyan, orange, blue, pink, and brown) and particle patterns. Particles were viewed from the bottom wall of the tank.
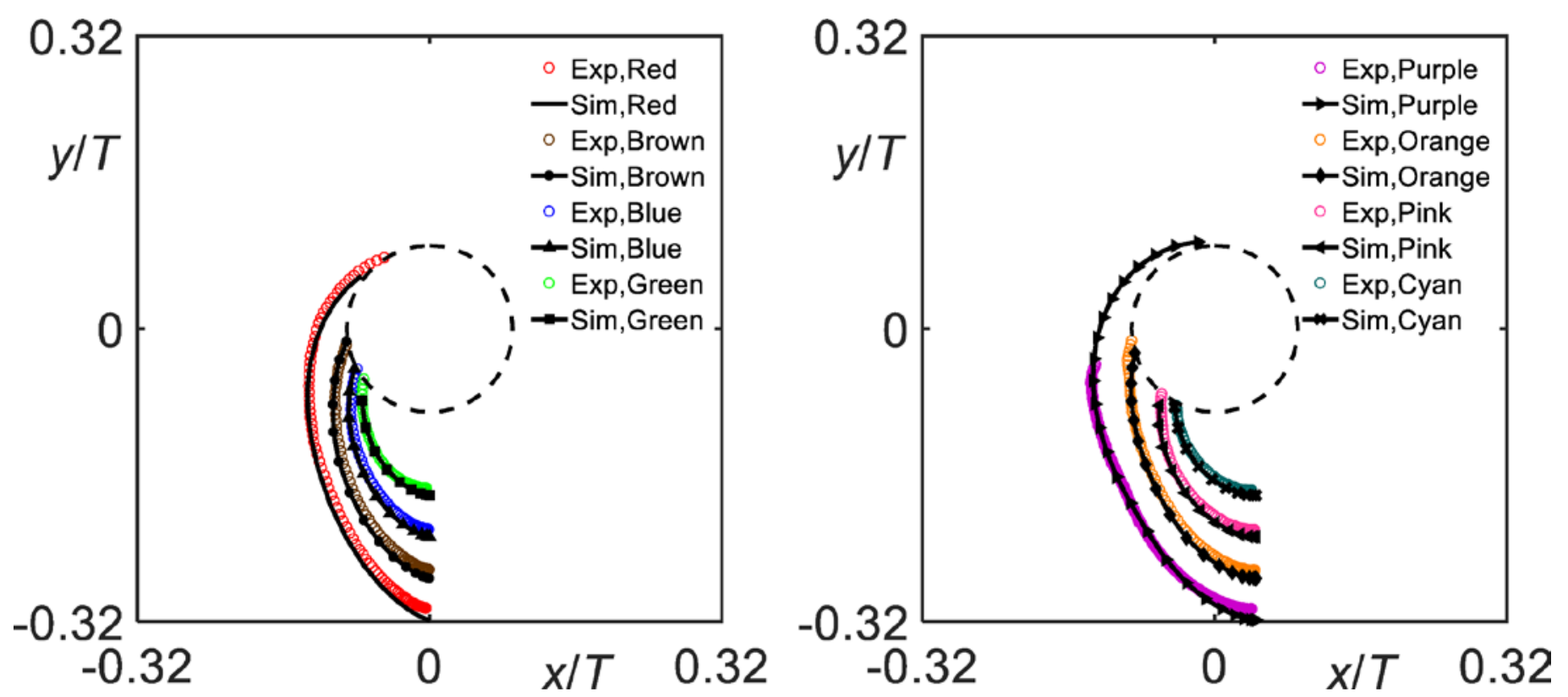

Fig. 11 - The experimental and simulated trajectories of eight particles rolling on the bottom wall. The legend in each panel contains data source and suspended particle represented by color. For example, "Sim" means simulated results, and "Red" is the color of the lift-off particle. A (dashed) circle with diameter of $4.0 d_{p}$ is used to avoid displaying some random particle trajectories near the bottom center.

The comparison between numerical and experimental results on the vertical locations and lift-off velocities of the multiple particles is shown in Fig. 12. The simulation does predict the slow increases of the vertical particle locations and associated velocity fluctuations shortly after $t=0$. This phenomenon is caused by particle's climbing up and rolling over other particles. As we mentioned before, randomness regarding contact point and local surface roughness of the particles makes the experimental results less reproducible (see Fig. 8). Thus, it is reasonable that there are some discrepancies between simulated results and experimental data at this stage (see Fig. 12). The simulated particle interaction at the early lift-off stages lasted a shorter period in comparison with the experimental ones for the first seven suspended particles, resulting in their velocity profiles slightly shifting to the left along the 
time axis. The simulated maximum velocities for the particles are in good agreement with the experimental results.

The two simulated velocity peaks of the purple particle were caused by the interaction with the green particle; the purple particle was lifted off very shortly after the lift-off of the green particle, and the latter affected the motion of the former, as shown in Fig. 9.
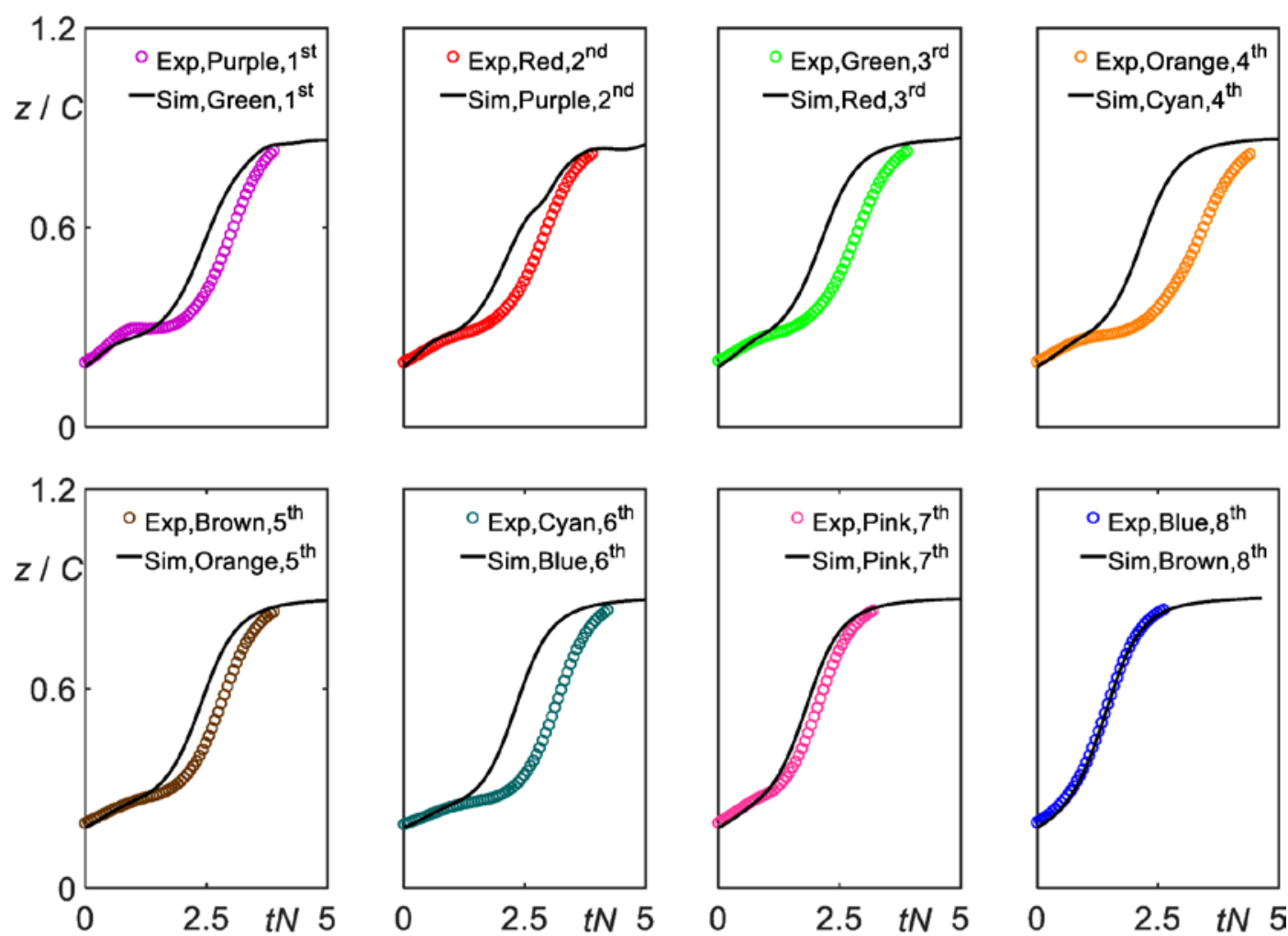

(a)
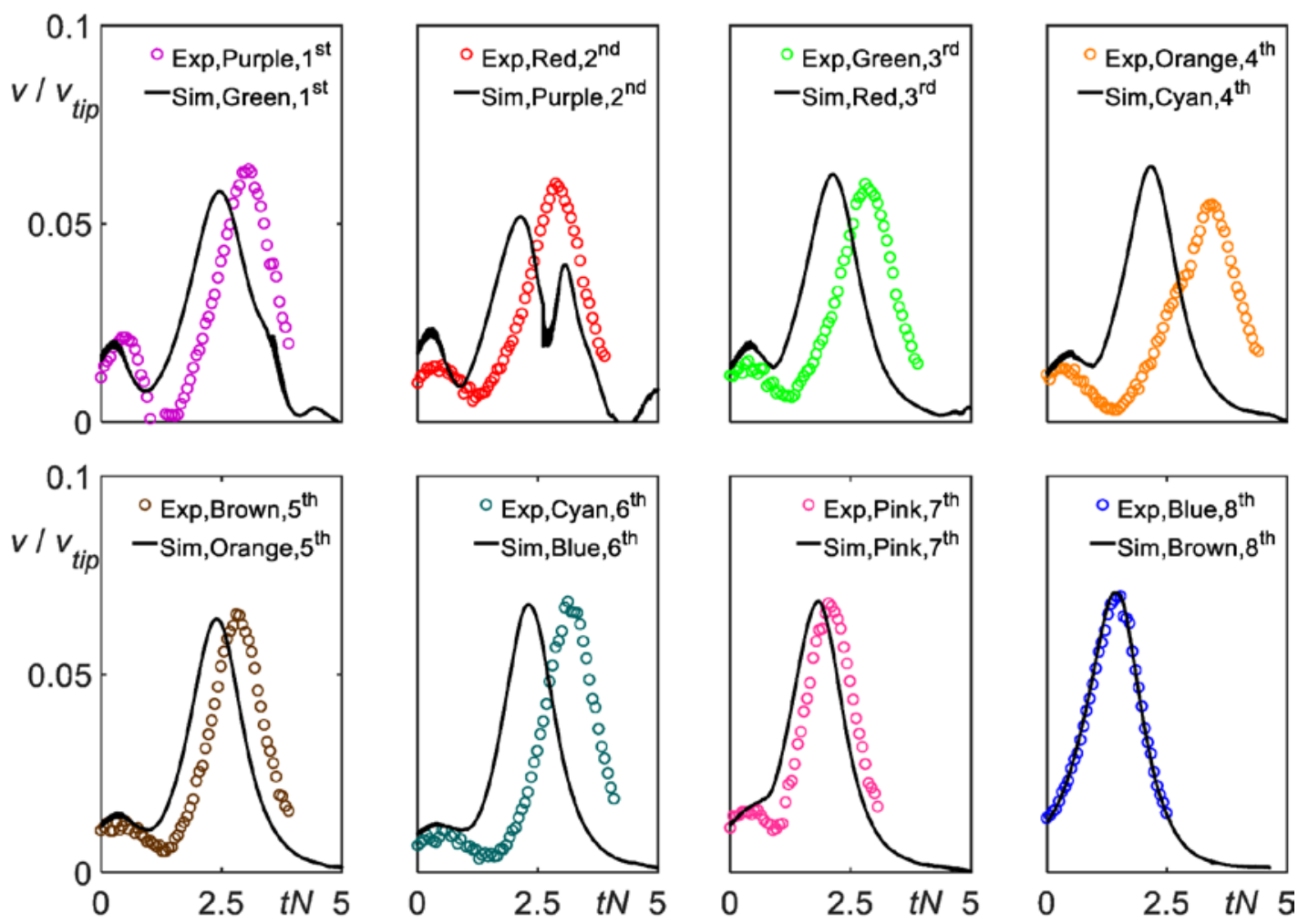

(b) 
Fig. 12 - Time series of the experimental and simulated particle vertical locations (a) and lift-off velocities (b) when the start times $t=0$ is set at $z=1.0 d_{p}$. The legend in each panel contains data source, suspended particle represented by color, and lift-off sequence. For example, "Sim" means simulated results, "Orange" is the color of the lift-off particle, and " 5 th" represents the fifth suspended particle.

The local flow information provided by the simulations helps to qualitatively understand the underlying cause of particle suspension. The liquid velocity vectors and the pressure underneath the impeller are given in Fig. 13. In our previous research (Mo et al., 2015) we found that the suspending force is proportional to the volume of the sphere and it scales as $F_{\text {susp }} \propto N^{1.4}$, which is in between viscous $\left(F_{\text {susp }} \propto N\right)$ and inertial $\left(F_{\text {susp }} \propto N^{2}\right)$ scaling. This result indicates that the pressure gradient force dominates the lift-off process. In this study, similar phenomena could be observed, including a low pressure region above the particle resting on the bottom, a laminar flow with a low Reynolds number, and a swirling liquid flow generated by a rotating impeller. Thus, we consider that the pressure gradient around the particles is a key factor for their initial lift-off. While the blue particle is climbing up other particles (see the top right panel of Fig. 13), the negative $\partial P / \partial z$ near the particle is still responsible for its lift-off. At the same time, the upward vertical flow around the particle also contributes to its suspension. With the rotation of the blade, the velocity and pressure fields behind the blade change a lot, but these changes do not have noticeable influence on the velocity and pressure distributions around the bottom center. 

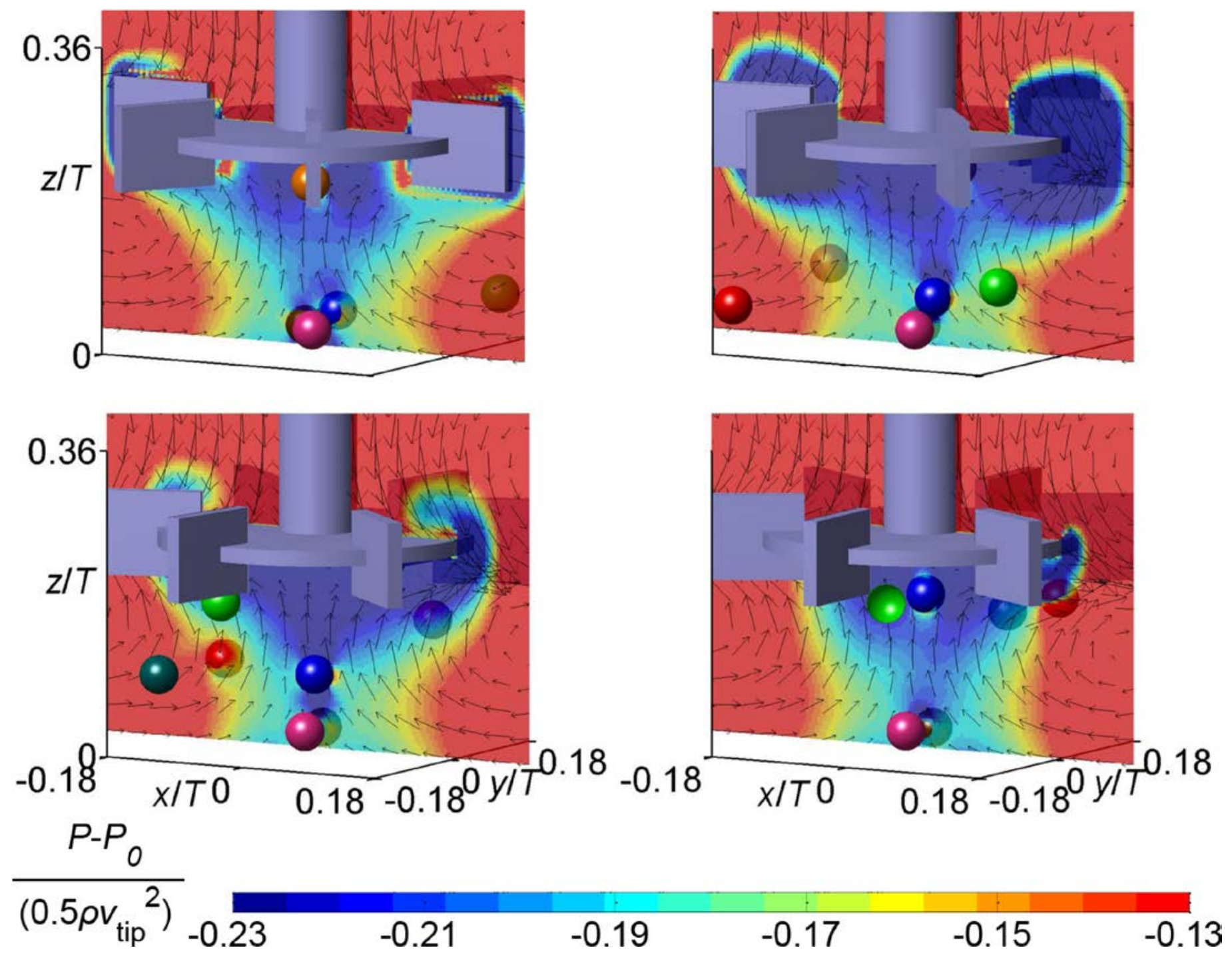

Fig. 13 - Normalized flow field and pressure distribution during the lift-off of the blue particle in the simulation.

\section{Conclusions}

Multi-particle suspension in a laminar stirred tank flow agitated by a standard Rushton turbine was investigated. This work is an extension of our previous research regarding a single particle (Mo et al., 2015). The diameter of the eight quartz particles is $10 \mathrm{~mm}$, and the impeller-based Reynolds number is about 19 . The particles motions at three stages, namely rolling over the tank bottom, interacting around the bottom center, and rising towards the turbine over the center line of the tank, were captured by two high-speed cameras. A matlab code was used to detect the time series of particle locations, and then the particle vertical velocities during the lift-off stage were calculated. 
The uncertainty of the measurement of the critical impeller speed $N_{\mathrm{LO}}$ is within $2 \%$ in the experiments. The $N_{\mathrm{LO}}$ increases with the increase of the number of suspended particles $n_{\llcorner O}$. Two stable particles patterns formed by three or four particles hinder the lift-off of unsuspended particles, and thus a significant speed increase is needed at $n_{\mathrm{LO}}=5$ and 8 to break the stable patterns. After the three-particle pattern is destroyed, all the three particles can be lifted off subsequently at the impeller speed. The triangle pattern with three particles could be considered as a more stable one because the phenomena $n_{\llcorner 0}=6$ and 7 did not happen in the experiments.

The trajectories of the particles rolling towards the bottom center are reproducible in the experiments, but the lift-off sequence and the pattern of the unsuspended particles present some random characteristics. Particle's interaction and local surface roughness at contact points might be the main reasons for the randomness. Partly reproducible results regarding particle vertical locations and velocities could be obtained if the origin of the time axis $t=0$ are set at the moment that particle center location $z$ is equal to $1.0 d_{p}$.

Direct numerical simulations based on the lattice Boltzmann method and the resolved particle model were performed, and the effect of friction coefficient and lubrication force model on multi-particle lift-off were evaluated. A friction coefficient of 0.1 provides the most reasonable simulated results. The use of the lubrication force model slows down the lift-off process. Typical radial flow pattern in a vertical plane and swirling flow pattern in a horizontal plane of the tank were obtained in the simulations. It determines the multi-particle motion at different stages. Overall, the simulated particle trajectories and velocities are in agreement with the experimental results, although there are some discrepancies in terms of particles lift-off sequence and at the early lift-off stage. The pressure gradient around particles is a key factor for their initial lift-off.

This work experimentally and numerically investigated the phenomena of multi-particle suspension in a simplified stirred tank. The validated simulation method will be used to enhance our understanding of particle suspension mechanisms as we move towards more complicated situations involving many more particles and/or transitional and turbulent flow. 


\section{Acknowledgements}

The financial supports from the National Key R\&D Program of China (2017YFB0306704) and the National Natural

Science Foundation of China (No.21676007) are gratefully acknowledged.

\section{Reference}

Agudo, J.R., Wierschem A., 2012. Incipient motion of a single particle on regular substrates in laminar shear flow. Phys. Fluids 24 , 093302.

Agudo, J.R., Dasilva, S., Wierschem, A., 2014. How do neighbors affect incipient particle motion in laminar shear flow? Phys. Fluids 26, 053303.

Atherton, T.J., Kerbyson, D.J., 1999. Size invariant circle detection. Image Vision Comput. 17, 795-803.

Ayranci, I., Machado, M.B., Madej, A.M., Derksen, J.J., Nobes, D.S., Kresta, S.M., 2012. Effect of geometry on the mechanisms for off-bottom solids suspension in a stirred tank. Chem. Eng. Sci. 79, 163-176.

Blais, B., Lassaigne, M., Goniva, C., Fradette, L., Bertrand, F., 2016. Development of an unresolved CFD-DEM model for the flow of viscous suspensions and its application to solid-liquid mixing. J. Comput. Phys. 318, 201-221.

Blais, B., Bertrand, F., 2017. CFD-DEM investigation of viscous solid-liquid mixing: Impact of particle properties and mixer characteristics. Chem. Eng. Res. Des. 118, 270-285.

Blais, B., Bertrand, O., Fradette, L., Bertrand, F., 2017. CFD-DEM simulations of early turbulent solid-liquid mixing: Prediction of suspension curve and just-suspended speed. Chem. Eng. Res. Des. 123, 388-406.

Carletti, C., Montante, G., Westerlund, T., Paglianti, A., 2014. Analysis of solid concentration distribution in dense solid-liquid stirred tanks by electrical resistance tomography. Chem. Eng. Sci. 119, 53-64.

Chen, S., Doolen, G.D., 2012. Lattice Boltzmann method for fluid flows. Annu. Rev. Fluid Mech. 30, 329-364.

Davies, E.R., 2005. Machine Vision: Theory, Algorithms, Practicalities, third ed. Morgan Kauffman Publishers, San Francisco.

Derksen, J.J., Van den Akker H.E.A., 1999. Large eddy simulations on the flow driven by a Rushton turbine. AIChE J. 45, $209-221$.

Derksen, J.J., 2011. Simulations of granular bed erosion due to laminar shear flow near the critical Shields number. Phys. Fluids 23, $113303-113314$.

Derksen, J.J., 2012. Highly resolved simulations of solids suspension in a small mixing tank. AIChE J. 58, 3266-3278.

Dilloo, M.J., Tangman, D.Y., 2017. A high-order finite difference method for option valuation. Comput. Math. Appl. 74, 652-670. Eggels, J.G.M., Somers, J.A., 1995. Numerical simulation of free convective flow using the lattice-Boltzmann scheme. Int. J. Heat Fluid FI. 16, 357-364.

Kee N.C.S., Tan R.B.H., 2002. CFD simulation of solids suspension in mixing vessels. Can J. Chem. Eng. 80, 721-726.

Kim, S., Karrila, S.J., 2005. Microhydrodynamics: Principles and Selected Applications, first ed. Dover Publications, New York. Lassaigne, M., Blais, B., Fradette, L., Bertrand, F., 2016. Experimental investigation of the mixing of viscous liquids and non-dilute concentrations of particles in a stirred tank. Chem. Eng. Res. Des. 108, 55-68. 
Li, G., Gao, Z., Li, Z., Wang, J., Derksen, J.J., 2018. Particle-resolved PIV experiments of solid-liquid mixing in a turbulent stirred tank. AIChE J. 64, 389-402.

Mo J., Gao, Z., Bao, Y., Li, Z., Derksen, J.J., 2015. Suspending a solid sphere in laminar inertial liquid flow - experiments and simulations. AIChE J. 61, 1455-1469.

Nguyen, N.Q., Ladd, A.J., 2002. Lubrication corrections for lattice-Boltzmann simulations of particle suspensions. Phys. Rev. E 66, 046708.

Somers, J.A., 1993. Direct simulation of fluid flow with cellular automata and the lattice-Boltzmann equation. Appl. Sci. Res. 51, 127-133.

Srinivasa T., Jayanti S., 2007. An Eulerian/Lagrangian study of solid suspension in stirred tanks. AIChE J. 53, $2461-2469$.

Succi, S., 2001. The Lattice Boltzmann Equation for Fluid Dynamics and Beyond, first ed. Clarendon Press, Oxford.

Tamburini, A., Cipollina, A., Micale, G., Brucato, A., Ciofalo, M., 2012. CFD simulations of dense solid-liquid suspensions in baffled stirred tanks: Prediction of the minimum impeller speed for complete suspension. Chem. Eng. J. 193-194, 234-255.

Ten Cate, A., Nieuwstad, C.H., Derksen, J.J., Van den Akker H.E.A., 2002. Particle imaging velocimetry experiments and lattice-Boltzmann simulations on a single sphere settling under gravity. Phys. Fluids 14, 4012-4025.

The MathWorks, Inc., 2017. Find circles using circular Hough transform.

http://cn.mathworks.com/help/images/ref/imfindcircles.html (accessed 20 Oct 2017).

Woo, J.J., Garaniya, V., Abbassi, R., 2016. Improving droplet sizing methodology for spray dynamics investigation. Int. J. Spray Combust. 8, 86-99.

Yamamoto, Y., Potthoff, M., Tanaka, T., Kajishima, T., Tsuji, Y., 2001. Large-eddy simulation of turbulent gas-particle flow in a vertical channel: effect of considering inter-particle collisions. J. Fluid Mech. 442, 303-334.

Ziegler, D.P., 1993. Boundary conditions for lattice Boltzmann simulations. J. Stat. Phys. 71, 1171-1177.

Zwietering, T.N., 1958. Suspending of solid particles in liquid by agitators. Chem. Eng. Sci. 8, 244-253. 